\title{
A THEORETICAL DETERMINATION OF THE CHARACTERISTIC EQUATION OF SNOW IN THE PENDULAR REGIME
}

\author{
By E.M. MORRIS \\ (British Antarctic Survey, Natural Environment Research Council, High Cross, Madingley Road, \\ Cambridge CB3 OET, England) \\ and R.J. KELLY
}

(School of Mathematics, University of East Anglia, Norwich NR4 7TJ, England)

\begin{abstract}
Recent mathematical models treat a natural snow-pack as a mixture body consisting of solid ice grains, liquid water, and a gas made up of air and water vapour. Such a model requires two independent constitutive equations for the two independent volume fractions. However, so far only one equation, a power law relating the liquid-water content to capillary pressure, has been suggested, by analogy with the so-called "characteristic" equation for liquid water in soils. Experimental data from drainage tests on snow columns may be used to determine the characteristic equation for snow for relatively high water contents. However, the experimental method is not valid when water exists in isolated inclusions in the snow, i.e. in the pendular regime. In this paper a theoretical method is used to derive two independent volume-fraction laws for snow in the pendular regime.
\end{abstract}

\section{INTRODUCTION}

In recent years, the continuum theory of mixture has been used as the framework for models of natural snow-packs (Morris, 1983; Akan, 1984; Kelly and others, 1986). The constitutive requirements for such a model are outlined in Kelly and others (1986). These authors pointed out that, if snow is treated as a three-constituent mixture of ice, liquid water and moist air, two volume-fraction equations are required to complete the model. Previous workers have identified only one such equation, an empirical power law relating water content to capillary pressure. This "characteristic" equation for snow is based on very limited data and only applies in conditions of moderate saturation. Thus, to establish a general model for natural snow-packs. it is necessary to extend the range of the empirical characteristic equation and, furthermore, to derive a second volume-fraction equation. This paper uses a theoretical analysis of snow-pack properties on the microscale to derive two volume-fraction equations for conditions of low water saturation, i.e. in the pendular regime.

The mixture theory adopted in the recent snow-melt models is that of Truesdell (1965). In this theory, the equations of conservation of mass, momentum, and energy for each constituent within the mixture are expressed in terms of "partial" variables associated with mixture volumes and mixture cross-section areas. Thus, for example, the partial pressure in a particular constituent is defined to be the force on that constituent per unit mixture crosssectional area. Models are completed by constitutive equations which may also involve "intrinsic" variables which are defined with respect to the volume and cross-section of a particular constituent. For a fully general model for snow, constitutive equations must be specified for the independent volume fractions, the independent phase changes, and for the permeabilities. These equations may be written in terms of partial or intrinsic variables, although it is a fundamental postulate of mixture theory that they should be determined from the gross response of the mixture body. Finally, stress-strain relations for each of the constituents within the mixture model are required. These may be written in terms of intrinsic variables and may be taken as the equations which represent the behaviour of the constituent as if it were an isolated continuum. There is therefore less difficulty in specifying these laws.

A simplification common to many snow models is to treat the air and water vapour as a single constituent. When this is done, a mixture model for snow will have three constituents: ice, water, and moist air. The volume fraction $\phi^{(\omega)}$ is defined as the volume of constituent $\omega$ per unit volume of the mixture. We write $\omega=1,2,3$ for ice, water, and moist air, respectively. By definition

$$
\sum_{\omega=1}^{3} \phi^{(\omega)}=1
$$

Thus, only two of the volume fractions are independent and only two independent constitutive equations need to be specified.

In the following discussion the intrinsic pressure $p^{(\omega)}$ will be introduced. It is important to establish exactly what is meant by this term in mixture theory. The intrinsic pressure of component $\omega$ on the mixture scale is the pressure that would be measured by an instrument which sampled the pressures of a large number of inclusions of $w$ and averaged them. For example, a tensiometer with a porous cup much larger than the grain-size of the snow would measure $p^{(2)}$. If the inclusions are interconnecting, they all have the same pressure and the intrinsic pressures on the microscopic and mixture-theory scales are the same.

\section{EXPERIMENTAL DATA}

Colbeck (1975) and Wankiewicz (1978) have used a simple moisture retention-curve apparatus to determine the characteristic equation for snow. It is worth describing the experimental method in some detail in order to clarify exactly what is being measured and assess the accuracy of the data. In the retention-curve apparatus a column of snow of volume $V$ rests on a porous plate through which water, but not air, can pass. The lower surface of the plate is in contact with water at a pressure $p_{\mathrm{w}}$. The applied capillary pressure, $\psi^{0}=p_{\mathrm{w}}-p_{\mathrm{a}}$ (where $p_{\mathrm{a}}$ is the atmospheric pressure), can be adjusted by altering the height of a 
reservoir. A change in height $\Delta h$ produces a change in applied capillary pressure $\Delta \psi^{0}=-\rho g \Delta h$ where $\rho$ is the density of water and $g$ is the acceleration due to gravity.

The amount of water $V_{w}$ which flows out of the snow sample when $\psi^{0}$ is changed is equal to the overflow from the reservoir, which can be measured. Thus, the change in average water content $\Delta \phi^{(2)}=V_{\mathrm{w}} / V$ can be determined as a function of $\Delta \psi^{0}$. A major assumption is now made, namely, that the intrinsic pressure on the mixture scale of all the water inclusions in the sample is equal to the water pressure applied to the porous plate. In this case, the capillary pressure of the water inclusions with respect to the outside atmosphere, $\psi^{*}=p^{(2)}-p_{a}$, is the same as the applied capillary pressure $\psi^{0}$ and $\Delta \phi^{(2)}$ is determined as a function of $\Delta \psi^{*}$.

In order to derive $\phi^{(2)}$ as a function of $\psi^{*}$, it is necessary to have a direct measurement of $\phi^{(2)}$ at one known value of $\psi^{*}$. Wankiewicz (1979) measured the residual water content at $\psi^{*}$ large and negative by a calorimetric method. Alternatively, the relative water saturation $S=\phi^{(2)} /\left(1-\phi^{(1)}\right)$ can be derived as a function of $\psi^{*}$ (Colbeck, 1975). Assuming that the snow sample is saturated at atmospheric pressure, the required known point is $S=1$ at $\psi^{*}=0$

Figure la shows the data given by Wankiewicz (1979).
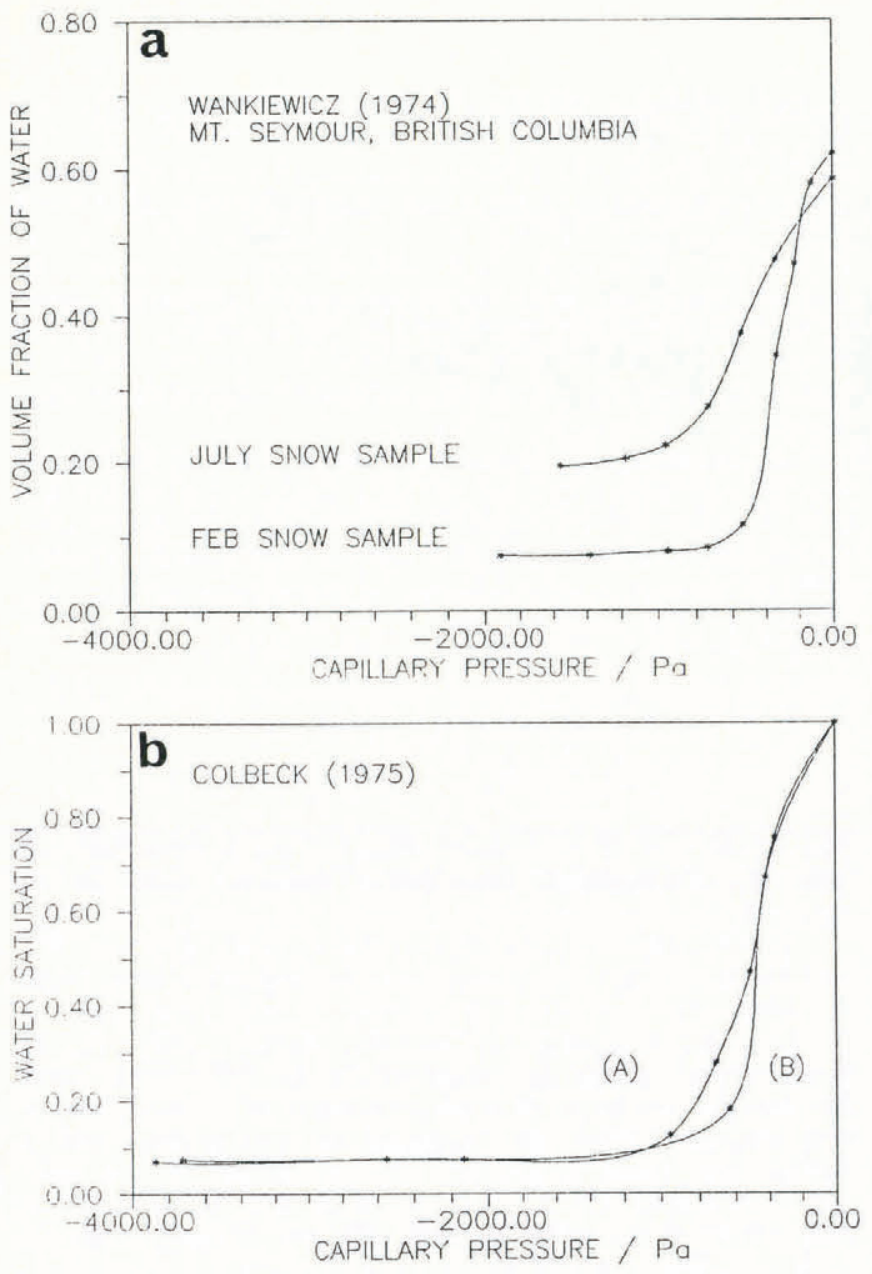

Fig. 1. Characteristic curves for snow derived from water-retention experiments. (a) Wankiewicz (1979), (b) Colbeck (1975).

His samples were taken from a natural snow-pack at or near the melting temperature. Figure lb shows the data given by Colbeck (1975). The axes have been scaled so the characteristic curves in (a) and (b) should be directly comparable. The curves have a form similar to those obtained by water-retention experiments on coarse-grained sand. The gradient changes between -600 and $-1000 \mathrm{~Pa}$ when water becomes trapped in the pores. Harris and
Morrow (1964) have found that this transition occurs at $\psi_{\mathrm{T}}=-10.5 \sigma_{23} / R_{13}$ for random packings of spheres of radius $R_{13}$. The interfacial tension between air and water $\sigma_{23}=0.075 \mathrm{~J} \mathrm{~m}^{-2}$ so that for $1 \mathrm{~mm}$ radius grains $\psi_{\mathrm{T}}=$ $-788 \mathrm{~Pa}$. A cubic packing of similar spheres would have $\psi_{\mathrm{T}}$ $=-362 \mathrm{~Pa}$ and a rhomboidal packing, $\rightarrow-968 \mathrm{~Pa}$. The concept of the irreducible water content of snow arises specifically from these water-retention experiments. The water content can of course go to zero in other situations, for example when heat is extracted from the snow sample.

Colbeck and Wankiewicz have drawn attention to the difficulties in determining the characteristic equation by this experimental method. Some of these problems also arise when working with soils but others are particular to snow. They may be summarized as follows:

(i) Each data point on the Wankiewicz curves has a potential error of $\Delta \phi^{(2)} \simeq \pm 0.02$ arising from the error in the calorimetric method of determination of the residual water content.

(ii) At applied capillary pressures below about $-1500 \mathrm{~Pa}$ the porous plate loses pressure contact with the bulk of the snow. Thus, $\psi^{0}$ is no longer equal to $\psi^{*}$ and the long flat tail of the characteristic curves shown in Figure 1 is an artefact of the experimental method.

(iii) Temperature control is obviously vital but it is extremely difficult to maintain truly adiabatic conditions.

Colbeck (1974) described retention experiments for snow in which the temperature-control problem was solved by using kerosene rather than water. However, these cannot be expected to give an accurate characteristic equation for water in snow. The volume of water at a given capillary pressure depends not only on the geometry of the ice grains (which can be maintained in the kerosene experiments) but also on the surface energies of the phase interfaces. These will not be the same in the ice-kerosene system.

De Quervain (1973) gave two water contents at different capillary pressures measured in a natural snowpack. The authors are not aware of any other published data for snow.

The experimental data were used by Morris and Godfrey (1979) to determine a characteristic equation for use in their snow-melt model. They postulated an equation of the form

$$
\phi^{(2)}=\left(1-\phi^{(1)}\right)\left(\psi / \psi_{\mathrm{a}}\right)^{s}
$$

where $\psi=p^{(2)}-p^{(3)}$ is the capillary pressure of the water inclusions with respect to the moist air inclusions. Equation (2) is one of the Brooks-Corey relationships (Brooks and Corey, 1964) used in modelling the flow of water in unsaturated soils. $\psi_{\mathrm{a}}$ is the "air-entry potential" or "bubbling pressure" and $s$ is an index of the geometry of the porous medium. Note that in the funicular regime, when the air inclusions are isolated by water and ice from the outside atmosphere, there is no reason why $p^{(3)}$ should equal $p_{\mathrm{a}}$. However, in the pendular regime of low water content, it is reasonable to assume that the air inclusions form an interconnecting network and $p^{(3)}=p_{\mathrm{a}}$. Hence $\boldsymbol{\psi}=\boldsymbol{\psi}^{*}$ in the pendular regime. The index $s$ and the air-entry potential $\psi_{a}$ were estimated using all the data from Colbeck (1975), Wankiewicz (1979), and de Quervain (1973) with the assumption that $\psi=\psi^{*}=\psi^{0}$ throughout the range. The values of $s=-0.93 \pm 0.12, \ln \left(-\psi_{\mathrm{a}} / \mathrm{N} \mathrm{m}^{-2}\right)=5.3 \pm 1.5$ have been used by Morris (1983) but clearly from the discussion above not all the experimental data points should have been used. If points with high and low water contents are discarded, new values of $s=-0.86$ and $\ln \left(-\psi_{\mathrm{a}} / \mathrm{N} \mathrm{m}^{-2}\right)=5.5$ are obtained (Fig. 2). These are not significantly different because of the large scatter on all the data.

\section{THEORETICAL DETERMINATION OF THE VOLUME- FRACTION EQUATIONS}

The primary aim of the theoretical work described in this paper has been to extend the range of the characteristic equation into the pendular regime. The analysis also gives a second volume-fraction equation valid in the pendular regime. This is a useful bonus. Previous models of unsaturated snow-packs have used Equation (2) as one of 


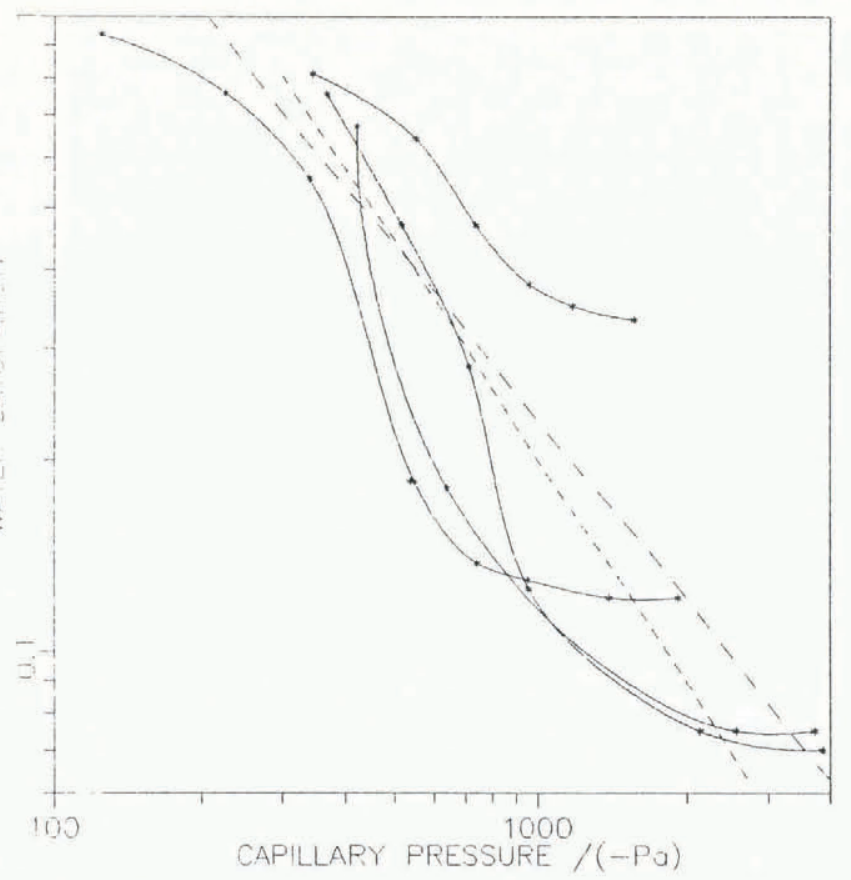

Fig. 2. The empirical power-law characteristic equation determined (a) using all data and (b) discarding data at high and low water contents. The solid lines show the experimental data.

the required relations and have avoided the problem of identifying a second relation by assuming that the ice-grain velocity is zero. However, this has the important consequence that compaction of the snow cannot be modelled. The identification of a second volume-fraction constitutive equation allows this important physical process to be included in a snow-pack model.

In order to make the theoretical calculations tractable, it is assumed that the ice grains and water in the snow exist in a simple, though still physically realistic geometry. It is supposed that all contacts between ice grains are either simple two-grain contacts with an annular water inclusion or three-grain contacts with three annular inclusions meeting at a vein as described by Colbeck (1979). The grains are assumed to be spherical, except at the ice-ice and ice-water interfaces. Following Gubler (1978), we define end grains with one contact (coordination number $n_{3}=1$ ), chain grains with $n_{3}=2$, and link or mesh grains with $n_{3}>2$.

We begin with the simple case of a collection of end or chain grains of equal radius and with two-grain contacts. The specific volumes of ice, water, and moist air may be calculated using the geometrical relations shown in Figure $3 a$, which shows the form of the phase interfaces. The geometry is symmetric about the line FO. For clarity, the figure is not drawn to scale. The radius of the ice-gas interface is $R_{13}=\mathrm{FB}=\mathrm{FN}$. The two radii of curvature of the ice-water interface are $R_{12}^{(1)}$ and $R_{12}^{(2)}$ where $R_{12}^{(1)}=\mathrm{DC}$ $=\mathrm{DB}$, and the average value of $R_{12}^{(2)}$ is given by IJ. The point $\mathrm{J}$ is situated such that the $\operatorname{arc} \mathrm{CJ}=\mathrm{BJ}$. Finally, $R_{23}^{(1)}$ and $R_{23}^{(2)}$ are the radii of the water-gas interface; $R_{23}^{(1)^{23}}=$ $\mathrm{AH}=\mathrm{AB}$ and $R_{23}^{(2)}=\mathrm{OH}$. The compressed sphere radius is $\delta=\mathrm{OF}$ and the length $\mathrm{ED}=a$.

The compression angle $\mathrm{OAF}=\phi$ is a measure of the size of the water inclusion and the compression of the grain. The angle $\alpha$ is the dihedral angle, which depends on the surface free energy of the ice-ice interface, $\sigma_{11}$. The value of $\alpha$ has been given as $20 \pm 10^{\circ}$ by Ketcham and Hobbs (1969) and this is the value used by Colbeck (1979). Walford and others (1987) have suggested a value of $\alpha=33.6 \pm 0.7^{\circ}$ but this is based on 14 measurements and he has subsequently revised the value downwards (personal communication). We expect the value of $\alpha$ to be dependent on the relative crystallographic orientations of the two ice grains. Values of $\alpha$ from $10^{\circ}$ to $60^{\circ}$ have been measured by Ketcham and Hobbs (1969).

With the above notation and definitions, it is a straightforward matter to evaluate the volume fractions in terms of the radii of curvature. These calculations are presented in Appendix 1.
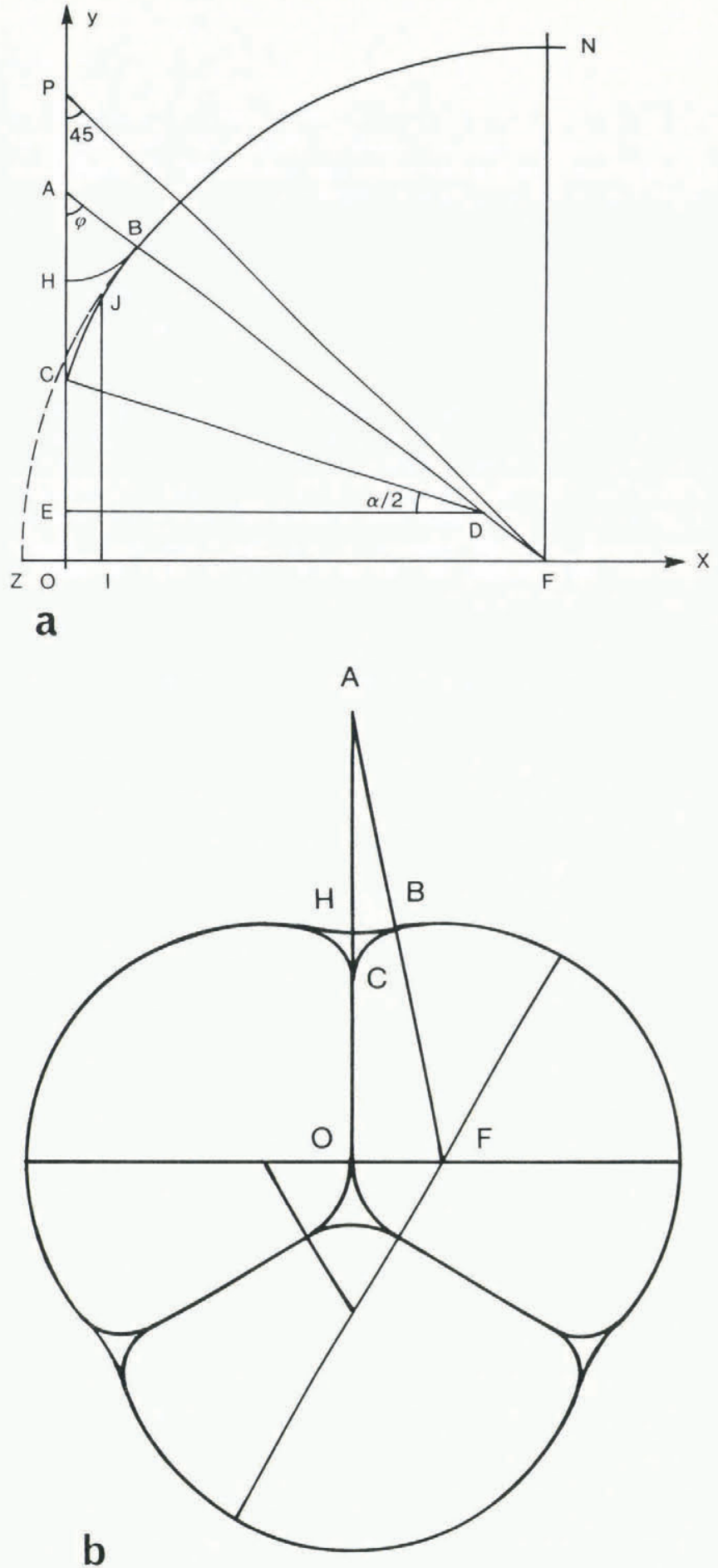

Fig. 3. Water inclusions at: (a) two-grain, and (b) three-grain intersections.

To express the volume fractions in terms of the intrinsic pressures, it is necessary to make a physical postulate, namely that the snow mixture is in mechanical equilibrium. In this case, the pressure difference across an interface between constituents is given in terms of the radii of curvature of the interface by the Laplace equations

$$
\begin{aligned}
& p^{(1)}-p^{(3)}=\sigma_{13}\left\{\begin{array}{l}
\frac{2}{R_{13}} \\
\}
\end{array}\right. \\
& p^{(2)}-p^{(3)}=\sigma_{23}\left\{\begin{array}{l}
\frac{1}{R_{23}^{(2)}}-\left|\frac{1}{\mathrm{R}_{23}^{(1)}}\right|
\end{array}\right\} \\
& p^{(1)}-p^{(2)}=\sigma_{12}\left\{\frac{1}{R_{23}^{(1)}}+\frac{1}{R_{12}^{(2)}}\right\}
\end{aligned}
$$


where $\sigma_{12}, \sigma_{13}$, and $\sigma_{23}$ are the interfacial tensions and are taken to be constants with respective values $0.034,0.109$, and $0.075 \mathrm{~J} \mathrm{~m}^{-2}$. Note that $\sigma_{12}+\sigma_{23}=\sigma_{13}$. This is required by the geometry which assumes that the contact angle of water on ice is zero. The negative sign in Equation (4) arises because of the saddle shape of the water-air interface. A relation between the various radii is implied by the three Laplace equations, namely

$$
\frac{2}{R_{13}} \sigma_{13}=\sigma_{12}\left\{\frac{1}{R_{12}^{(1)}}+\frac{1}{R_{12}^{(2)}}\right\}+\sigma_{23}\left\{\frac{1}{R_{12}^{(2)}}-\left|\frac{1}{R_{23}^{(1)}}\right|\right\}
$$

Using the Laplace equations and the geometrical relations established in Appendix 1, it is possible to calculate the volume fractions as a function of the pressure difference $\psi$, the ice-grain radius $R_{13}$, the coordination number $n_{3}$ (in this case the number of two-grain contacts per grain), and the number of ice grains per unit volume $N$. The dry density of the snow, $\rho_{\mathrm{s}}$, is also defined in terms of these variables since

$$
\rho_{\mathrm{S}}=\rho_{\mathrm{i}} \phi^{(1)}=\rho_{\mathrm{i}}\left(1-\phi^{(2)}-\phi^{(3)}\right) \text {. }
$$

The compression required to achieve mechanical equilibrium limits the number of simple two-grain contacts per grain. For example, three two-grain contacts will interfere unless $\sin \phi>0.5$, four contacts will interfere unless $\sin \phi>0.707$, and so on. These high values of $\sin \phi$ are only found with very low water contents. So, for link grains with $n_{3}>2$, three-grain intersections must be taken into account. In Appendix 2, expressions for the volume fractions are derived in terms of the radii of curvature for a system of grains of equal radius and contacts between two or between three grains. In this case, the water is held in pendular rings and in veins at three-grain intersections (Fig. 3b).

Equations (3)-(6) may also be applied to this system. Although more complex clusters of grains undoubtedly occur, they will not be included in the model at this stage. In addition, the model does not include water trapped in pores. It thus represents the situation during wetting rather than drying of the snow.

\section{RESULTS}

In order to facilitate comparison with the experimental data, the two volume-fraction curves are presented in terms of the water saturation $S=\phi^{(2)} /\left(1-\phi^{(1)}\right)$ and the (dry) density $\rho_{S}=\rho_{\mathrm{i}} \phi^{(1)}$ rather than $\phi^{(1)}$ and $\phi^{(2)}$. Figure $4 \mathrm{a}$ shows $S$ as a function of $\psi$ for a density of $300 \mathrm{~kg} \mathrm{~m}^{-3}$, $n_{3}=2$, and ice-grain radii varying from 0.01 to $1 \mathrm{~mm}$. The solid lines show the water saturation in the pendular regime calculated using the theoretical analysis of the previous section and the Appendices; the best straight line through the experimental data in the pendular-funicular transition region (E) is shown for comparison. The compression angle $\phi$ varies from $11.5^{\circ}$ at the high saturation end of each theoretical curve to $17.5^{\circ}$ at the low saturation end. Figure $4 \mathrm{~b}$ shows the curves for $0.1 \mathrm{~mm}$ grain radius, $n_{3}=2$, and densities varying from 100 to $500 \mathrm{~kg} \mathrm{~m}^{-3}$. Figure $4 \mathrm{c}$ shows the curves for density $500 \mathrm{~kg} \mathrm{~m}^{-3}, 1 \mathrm{~mm}$ grain radius, and coordination numbers from 1 to 4 . There are two curves for $n_{3}=2$, the lower one for a structure with two-grain intersections per grain and the upper one for a structure with one three-grain intersection per grain. For clarity, Figure 4 shows the effect of varying dry density, grain-size, or coordination number while holding the other two parameters constant. This establishes the form of the characteristic curve over a field of values. However, it should be noted that not all combinations of parameter

Fig. 4. Water saturation $S$ as a function of capillary pressure $\psi$ in the pendular regime (a) $\rho_{S}=300 \mathrm{~kg} \mathrm{~m}^{-3}$, $n_{3}=2$, (b) $R_{13}=0.1 \mathrm{~mm}, n_{3}=2$, (c) $\rho_{s}=500 \mathrm{~kg} \mathrm{~m}^{-3}$. $R_{13}=1 \mathrm{~mm}$.
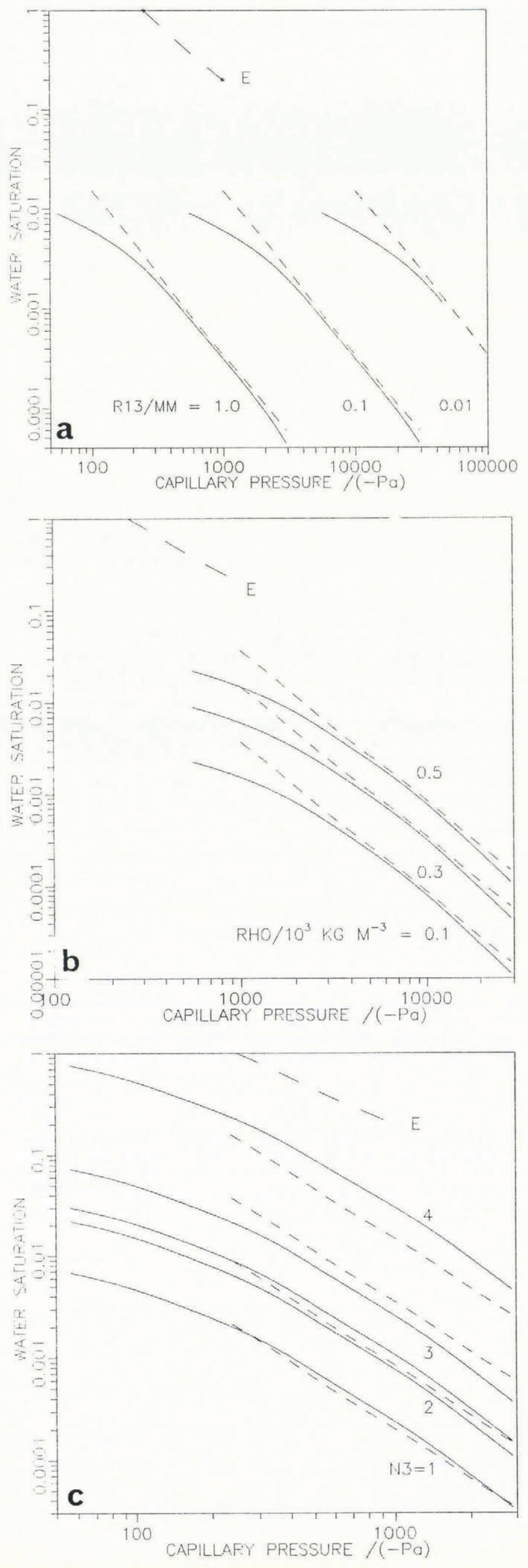
values within the field will be found in real snow. For example, in real snow $n_{3}$ will not be independent of $\rho_{\mathrm{S}}$.

Although the numerical scheme to calculate the characteristic curve is fairly simple and can easily be incorporated in a snow model, there will be occasions when it is useful to have an approximate analytical expression. The dashed lines in Figure 4 have been calculated using Equation (2), with best values of the parameters $s$ and $\psi_{\mathrm{a}}$ determined from the theoretical curves. For the ranges $\rho_{\mathrm{S}}=300-500 \mathrm{~kg}$ $\mathrm{m}^{-3}, R_{13}=0.01-1.0 \mathrm{~mm}$, and $n_{3}=1-4$

$$
\phi^{(2)}=\left(1-\phi^{(1)}\right)\left(\psi / \psi_{\mathrm{a}}\right)^{s}
$$

with

$$
s=-1.64 \pm 0.02,
$$

and $\quad \psi_{\mathrm{a}}=\left(-10^{-3} \mathrm{~Pa} \mathrm{~m} / R_{13}\right)\left(3.34 \exp \left(1.43 n_{3}\right) \rho_{\mathrm{S}} /\left(\rho_{\mathrm{i}}-\rho_{\mathrm{S}}\right)\right)^{0.61}$.

The dry density varies approximately as

$$
\rho_{\mathrm{S}}=\frac{4 \pi}{3}\left(R_{13}\right)^{3} N \rho_{\mathrm{i}}\left(1-0.240 n_{3}\right) \text {. }
$$

There is a weak dependence on $\left(\psi R_{13}\right)$ but this can be neglected. Equation (9) implies that each sphere of ice loses approximately $24 \%$ of its volume when it is compressed at a contact. It follows that the equation cannot be extrapolated to $n_{3}>4$. This is consistent with the initial assumption of non-interfering two- or three-grain contacts which restricts the number of contacts possible per grain. Equations (8) and (9) can be used as the two volume-fraction equations for snow with uniform grain-size and coordination number. The three fundamental variables describing the nature of the snow are the grain-size, coordination number, and number of grains per unit volume. It is assumed that all these can be measured by stereological methods.

The next stage is to define the range of grain-sizes and coordination numbers in a natural snow sample and calculate the appropriate volume-fraction equations. Let the grain-size distribution be given by a log-normal distribution (Colbeck, 1986)

$$
f\left(\ln R_{13}\right)=\frac{1}{(2 \pi)^{\frac{1}{2}} \ln \sigma_{\mathrm{g}}} \exp \left\{-\frac{1}{2}\left\{\frac{\ln \left(R_{13} / \bar{R}_{13}\right)}{\ln \sigma_{\mathrm{g}}}\right\}^{2}\right\}
$$

Colbeck (1986) has tabulated $\sigma_{\mathrm{g}}$ and $\bar{R}_{13}$ for some saturated snow samples. Mean radius varies from 0.1 to $1 \mathrm{~mm}$ and $\sigma_{\mathrm{g}}$ is 1.4-1.6. The mean grain-size of new dry snow can be much smaller. The probability of finding a grain with diameter less than $R_{13}$ is

$$
F\left(\ln R_{13}\right)=\int_{-\infty}^{\ln R_{13}} f(\ln R) \mathrm{d}(\ln R) .
$$

It will be assumed that, when a grain of diameter $R_{13}$ is in contact with another grain of smaller diameter, it produces a "size $R_{13}$ " inclusion, that is, an inclusion with the size, shape, and capillary pressure that would be produced between two grains of radius $R_{13}$. This simple postulate implies that the smaller grain is not in mechanical equilibrium. Colbeck (1979) has pointed out that ice grains of different sizes in a cluster develop grain boundaries which are curved so that mechanical equilibrium is restored. The smaller grains intrude into the space of the larger grains. At this stage, it is not appropriate to introduce such geometric complexity into the model.

Let the probability of a grain having $n_{3}$ contacts be given by Gubler's (1978) equation

$$
p\left(n_{3}\right)=\frac{4 i \alpha^{3 / 2}}{\pi^{1 / 2}}\left(n_{3}\right)^{(3 i-1)} \exp \left(-\alpha\left(n_{3}\right)^{2 i}\right) .
$$

$\alpha$ and $i$ are constants. Gubler $\left(1978\right.$, fig. 5) has shown $p\left(n_{3}\right)$ for $i=1.5$ and $\alpha=0.07636$. The mean coordination number is then $\bar{n}_{3}=2.5$, a typical value, although $\bar{n}_{3}$ varied in his samples from 2 to 8 . Note that it is assumed that $p\left(n_{3}\right)$ is independent of $R_{13}$.

The number of inclusions of size $R_{13}$ in contact with grains of radius $R_{13}$ is

$$
\int_{0}^{\infty} p\left(n_{3}\right) n_{3} F\left(\ln R_{13}\right) \mathrm{d} n_{3}=\bar{n}_{3} F\left(\ln R_{13}\right)
$$

where $\bar{n}_{3}$ is the average number of contacts per grain. The total number of size $R_{13}$ inclusions is $N f\left(\ln R_{13}\right) \bar{n}_{3} F\left(R_{13}\right)$ and the total number of inclusions is

$$
\int_{-\infty}^{\infty} N f\left(\ln R_{13}\right) \bar{n}_{3} F\left(\ln R_{13}\right) \mathrm{d}\left(\ln R_{13}\right)=N \bar{n}_{3} \int_{0}^{1} F \mathrm{~d} F=N \bar{n}_{3} / 2 .
$$

Normalizing Equation (13) gives the distribution function for inclusions of size $R_{13}$

$$
q\left(\ln R_{13}\right)=2 f\left(\ln R_{13}\right) F\left(\ln R_{13}\right) .
$$

Figure 5 shows normalized probability densities $p(X)$ and $q(X)$ (in units of $2(2)^{\frac{1}{2}} \ln \sigma_{\mathrm{g}}$ ) for grain-size and inclusion size. The normalized parameter $X=\ln \left(R_{13} / \bar{R}_{13}\right) /\left((2)^{\frac{1}{2}} \ln \sigma_{\mathrm{g}}\right)$. As would be expected from the assumption that the larger of a pair of grains controls the inclusion size, the peak of $q(X)$ is shifted to positive values of $X$.

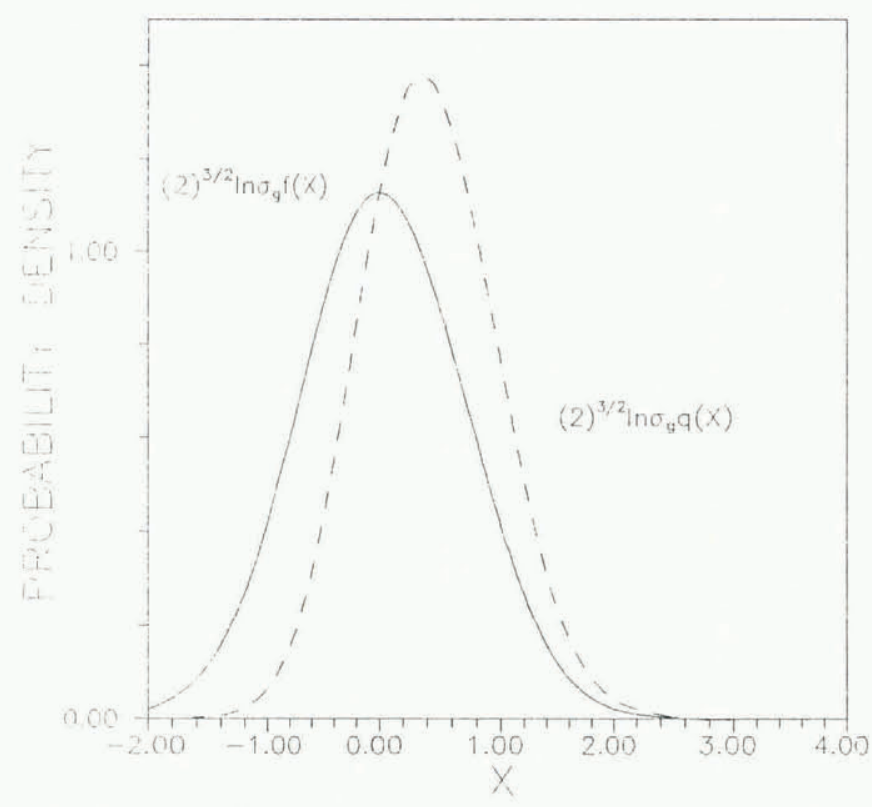

Fig. 5. Normalized probabilities $f(X)$ for grain-size and $q(X)$ for inclusion size.

The volume fractions are weighted as follows:

$$
\begin{aligned}
& \phi^{(2)}=\int_{0}^{\infty} \int_{-\infty}^{\infty} \phi^{(2)} q \mathrm{~d}\left(\ln R_{13}\right) p \mathrm{~d} n_{3} \\
& \Phi^{(1)}=\int_{0}^{\infty} \int_{-\infty}^{\infty} \phi^{(1)} f \mathrm{~d}\left(\ln R_{13}\right) p \mathrm{~d} n_{3} .
\end{aligned}
$$

In terms of the saturation and density for a uniform sample of $N$ grains, size $\bar{R}_{13}$ and coordination number $\bar{n}_{3}$, the two volume-fraction laws may be written

$$
\begin{gathered}
\bar{S}=\left[\psi / \Psi_{\mathrm{a}}\left(N, \bar{R}_{13}, \bar{n}_{3}\right)\right]^{S} K_{1}\left(\sigma_{\mathrm{g}}\right) K_{2}(\alpha, i), \\
\bar{\rho}_{\mathrm{S}}=\rho_{\mathrm{S}}\left(N, \bar{R}_{13}, \bar{n}_{3}\right) K_{3}\left(\sigma_{\mathrm{g}}\right)
\end{gathered}
$$

where the factors $K_{1}, K_{2}$, and $K_{3}$ show the effect of variability in the sample and may be calculated from Equations (15) and (16). Table I shows some typical values.

It is apparent that the effect of having a mixture of grain-sizes and coordination numbers is to increase the 


$\begin{array}{ccc}\sigma_{\mathrm{g}} & K_{1} & K_{3}=\exp \left(4.5 \ln ^{2} \sigma_{\mathrm{g}}\right) \\ 1.5 & 1.565 & 2.096 \\ 2.0 & 1.424 & 8.689 \\ 2.5 & 1.726 & 43.73 \\ 3.0 & 2.171 & 228.4\end{array}$

liquid-water content by a factor of 3-4 above that expected for a uniform sample with the same average properties. Figure 6 shows some example characteristic curves for snow mixtures with $\sigma_{\mathrm{g}}=1.5, i=1.5, \bar{n}_{3}=2.5, \bar{R}_{13}=0.1 \mathrm{~mm}$, and dry density varying from 100 to $500 \mathrm{~kg} \mathrm{~m}^{-3}$. These wetting curves are now closer to the empirical (drying) curve although still lie below it, as they should, since there is hysteresis in the drying curve.

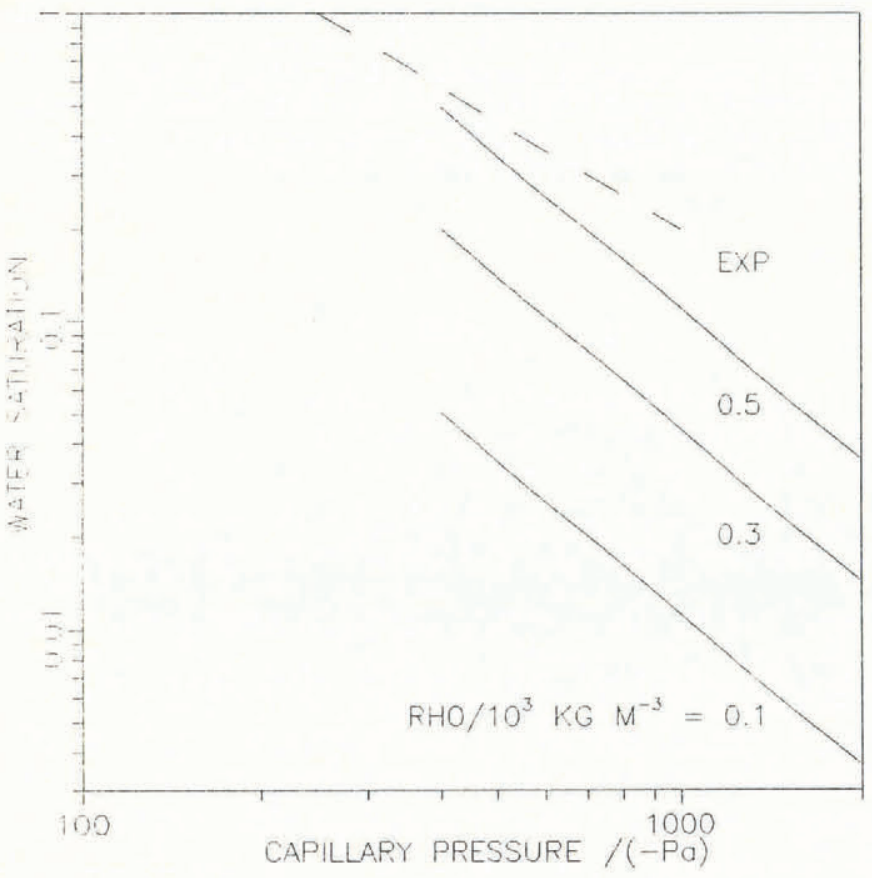

Fig. 6. Water saturation $\bar{S}$ as a function of capillary pressure in the pendular regime for snow with average grain-size $\bar{R}_{13}=0.1 \mathrm{~mm}$, average coordination number $\bar{n}_{3}=2.5, \sigma_{g}=1.5, \alpha=0.07636$, and $i=1$.

\section{CONCLUSION}

By assuming that the water and ice exist in a particular geometry, we have established, by theoretical means, the two volume-fraction constitutive laws required by three-component mixture models of snow. Thus, for the first time, it is possible to specify a complete threecomponent mixture model of snow in which the ice-grain velocity is not set to zero and compaction is allowed. However, by specifying a micro-geometry, we are, in a sense, contradicting the philosophical approach of general mixture theory. Mixture theory requires that experiments to determine the constitutive equations should be applied to the mixture as a whole. Clearly, calculations based on simplified geometric assumptions cannot necessarily be expected to give correct results concerning the gross response. There is an urgent need for more experimental data. The advantage of the micro-physical approach is that it is a means, at least in the short term, of completing a general three-phase model which includes all the important physical processes.

\section{ACKNOWLEDGEMENTS}

Part of the work reported in this paper was carried out while R.J. Kelly was supported by a U.K. Natural Environment Research Council Case Studentship, in

$\begin{array}{cccc}\alpha & i & \bar{n}_{3} & K_{2} \\ 0.07636 & 1.5 & 2.5 & 1.959 \\ 0.02959 & 2.0 & 2.5 & 1.442 \\ 0.35395 & 1.5 & 1.5 & 1.383\end{array}$

colloboration with Professor L.W. Morland. We are also grateful to $\mathrm{Dr} \mathrm{H}$. Gubler and other members of the Swiss Federal Institute for Avalanche Research for useful discussions.

\section{REFERENCES}

Akan, A. 1984. Simulation of runoff from snow covered hill slopes. Water Resour. Res., 20(6), 707-713.

Brooks, R.H. and A.T. Corey. 1964. Hydraulic properties of porous media. Fort Collins, $\mathrm{CO}$, Department of Civil Engineering, Colorado State University. (Hydrology Paper No. 3.)

Colbeck, S.C. 1974. The capillary effects on water percolation in homogeneous snow. J. Glaciol., 13(67), 85-97.

Colbeck, S.C. 1975. Grain and bond growth in wet snow. International Association of Hydrological Sciences Publication 114 (Symposium at Grindelwald 1974 - Snow Mechanics), 51-61.

Colbeck, S.C. 1979. Grain clusters in wet snow. J. Colloid Interface Sci., 72(3), 371-384.

Colbeck, S.C. 1986. Statistics of coarsening in watersaturated snow. Acta. Metall., 34(3), 347-352.

Gubler, H. 1978. Determination of the mean number of bonds per snow grain and of the dependence of the tensile strength of snow on stereological parameters. $J$. Glaciol., 20(83), 329-341.

Harris, C.C. and N.R. Morrow. 1964. Pendular moisture in packings of equal spheres. Nature, 203, 706-708.

Kelly, R.J., L.W. Morland and E.M. Morris. 1986. A three phase mixture model for melting snow. International Association of Hydrological Sciences Publication 155 (Symposium at Budapest 1986 - Modelling SnowmeltInduced Processes), 17-26.

Ketcham, W.M. and P.V. Hobbs. 1969. An experimental determination of the surface energies of ice. Philos. Mag., 19(162), 1161-1173.

Morris, E.M. 1983. Modelling the flow of mass and energy within a snowpack for hydrological forecasting. Ann. Glaciol., 4, 198-203.

Morris, E.M. and J. Godfrey. 1979. The European hydrological system snow routine. In Colbeck, S.C. and M. Ray, eds. Proceedings of a Meeting on Modeling of Snow Cover Runoff ... 1978, Hanover, New Hampshire ... Hanover, NH, US Army Cold Regions Research and Engineering Laboratory, 269-278.

Nye, J.F. 1989. The geometry of water veins and nodes in polycrystalline ice. J. Glaciol., 35(119), 17-22.

Quervain, M.R. de. 1973. Snow structure, heat, and mass flux through snow. International Association of Hydrological Sciences Publication 107 (Symposium at Banff 1972 - Role of Snow and Ice in Hydrology), 203-226.

Trusdell, C. 1965. The rational mechanics of materials. In On the foundations of mechanics and energetics. New York, Gordon and Breach, 293-304.

Walford, M.E.R., D.W. Roberts and I. Hill. 1987. Optical measurements of water lenses in ice. J. Glaciol., 33(114), 159-161.

Wankiewicz, A. 1978. Water pressure in ripe snowpacks. Water Resour. Res., 14(4), 593-600.

Wankiewicz, A. 1979. A review of water movement in snow. In Colbeck, S.C. and M. Ray, eds. Proceedings of a Meeting on Modeling of Snow Cover Runoff ... 1978, Hanover, New Hampshire ... . Hanover, NH, US Army Cold Regions Research and Engineering Laboratory, 222-252. 


\section{APPENDIX 1}

In this Appendix, expressions for the volume fractions $\phi^{(2)}$ and $\phi^{(3)}$ in the pendular regime are derived for the simple system in which all ice grains are of equal radius and all contacts are between two grains only, with the geometry shown in Figure $3 \mathrm{a}$. In this case, the coordination number, $n_{3}$, is equal to the number of two-grain contacts per grain, $n_{1}$. Let the volume obtained by rotating curve $\mathrm{HB}$ around the $x$-axis be denoted by VHB, and so on. Then the volume of ice in unit volume of snow is

$$
\phi^{(1)}=N\left(2 \mathrm{VZN}-n_{3}(\mathrm{VZB}-\mathrm{VCB})\right)
$$

where $N$ is the number of grains per unit volume, (VZB - VCB) is the volume of ice lost by a particular grain because of the formation of a two-grain contact, and $2 \mathrm{VZN}$ is the volume of a spherical grain with no contacts, i.e.

$$
\mathrm{VZN}=\frac{2 \pi}{3}\left(R_{13}\right)^{3}
$$

The specific volume of water is

$$
\phi^{(2)}=N n_{3}(\mathrm{VHB}-\mathrm{VCB})
$$

and the specific volume of moist air is

$$
\phi^{(3)}=1-N\left(2 \mathrm{VZN}-n_{3}(\mathrm{VZB}-\mathrm{VHB})\right) .
$$

The volumes VCB, VHB, and VZB are calculated as follows: with $x$ - and $y$-axes set up as in Figure $3 a$, the equation of the curve $Z B$ is

$$
y^{2}+\left(x-x_{\mathrm{F}}\right)^{2}=\left(R_{13}\right)^{2}
$$

where $x_{F}, y_{F}$ are the coordinates of point $\mathrm{F}$.

$$
x_{\mathrm{F}}=\delta \quad x_{\mathrm{Z}}=\delta-R_{13} .
$$

Hence

$$
\begin{aligned}
\mathrm{VZB}= & \pi \int_{x_{\mathrm{Z}}}^{x_{\mathrm{B}}}\left[\left(R_{13}\right)^{2}-\left(x-x_{\mathrm{F}}\right)^{2}\right] \mathrm{d} x \\
= & \pi\left[x_{\mathrm{B}}\left(\left(R_{13}\right)^{2}-\delta^{2}\right)-\left(x_{\mathrm{B}}\right)^{3} / 3+\left(x_{\mathrm{B}}\right)^{2} \delta-\left(\delta-R_{13}\right)\left(\left(R_{13}\right)^{2}-\delta^{2}\right)+\right. \\
& \left.+\left(\delta-R_{13}\right)^{3} / 3-\left(\delta-R_{13}\right)^{2} \delta\right] .
\end{aligned}
$$

The equation of the curve $\mathrm{CB}$ is

$$
\left(y-y_{\mathrm{D}}\right)^{2}+\left(x-x_{\mathrm{D}}\right)^{2}=\left(R_{12}^{(1)}\right)^{2}
$$

where

$$
x_{\mathrm{D}}=a \quad y_{\mathrm{D}}=\left(R_{13}-R_{12}^{(1)}\right) \cos \phi .
$$

Hence

$$
\begin{aligned}
\mathrm{VCB}= & \pi \int_{0}^{x_{\mathrm{B}}}\left[y_{\mathrm{D}}+\left[\left(R_{12}^{(1)}\right)^{2}-\left(x-x_{\mathrm{D}}\right)^{2}\right]^{\frac{1}{2}}\right]^{2} \mathrm{~d} x \\
= & \pi\left[x_{\mathrm{B}}\left(\left(R_{13}-R_{12}^{(1)}\right)^{2} \cos ^{2} \phi+\left(R_{12}^{(1)}\right)^{2}-a^{2}\right)-\left(x_{\mathrm{B}}\right)^{3} / 3+\left(x_{\mathrm{B}}\right)^{2} a\right]+ \\
& +\pi\left(R_{13}-R_{12}^{(1)}\right) \cos \phi\left[\left(x_{\mathrm{B}}-a\right)\left(\left(R_{12}^{(1)}\right)^{2}-\left(x_{\mathrm{B}}-a\right)^{2}\right)^{\frac{1}{2}}+\right. \\
& \left.+\left(R_{12}^{(1)}\right)^{2} \sin ^{-1}\left(\left(x_{\mathrm{B}}-a\right) / R_{12}^{(1)}\right)+a\left(\left(R_{12}^{(1)}\right)^{2}-a^{2}\right)^{\frac{1}{2}}-\left(R_{12}^{(1)}\right)^{2} \sin ^{-1}\left(-a / R_{12}^{(1)}\right)\right] .
\end{aligned}
$$

The equation of the curve $\mathrm{HB}$ is

$$
\left(y_{\mathrm{A}}-y\right)^{2}+x^{2}=\left(R_{23}^{(1)}\right)^{2}
$$

where

$$
y_{\mathrm{A}}=R_{23}^{(2)}+R_{23}^{(1)} \text {. }
$$


Hence

$$
\begin{aligned}
\mathrm{VHB}= & \pi \int_{0}^{x_{\mathrm{B}}}\left[y_{\mathrm{A}}-\left[\left(R_{23}^{(1)}\right)^{2}-x^{2}\right]^{\frac{1}{2}}\right]^{2} \mathrm{~d} x \\
= & \pi\left[x_{\mathrm{B}}\left(\left(R_{23}^{(2)}+R_{23}^{(1)}\right)^{2}+\left(R_{23}^{(1)}\right)^{2}\right)-\left(x_{\mathrm{B}}\right)^{3} / 3\right]- \\
& -\pi\left(R_{23}^{(2)}+R_{23}^{(1)}\right)\left[x_{\mathrm{B}}\left(\left(R_{23}^{(1)}\right)^{2}-\left(x_{\mathrm{B}}\right)^{2}\right)^{\frac{1}{2}}+\left(R_{23}^{(1)}\right)^{2} \sin ^{-1}\left(x_{\mathrm{B}} / R_{23}^{(1)}\right)\right] .
\end{aligned}
$$

The following geometric relations hold:

$$
\begin{aligned}
a & =\sin \phi\left(R_{23}^{(1)}+R_{12}^{(1)}\right) \\
\delta & =\sin \phi\left(R_{13}+R_{23}^{(1)}\right) \\
a & =\cos \frac{\alpha}{2} R_{12}^{(1)} \\
\delta^{2}+\left(R_{23}^{(2)}+R_{23}^{(1)}\right)^{2} & =\left(R_{23}^{(1)}+R_{13}\right)^{2} \\
R_{12}^{(2)} & =\left(R_{13}-R_{12}^{(1)}\right) \cos \phi+R_{12}^{(1)} \sin \left(\frac{\pi}{4}-\frac{\phi}{2}+\frac{\alpha}{4}\right) \\
x_{\mathrm{B}} & =\sin \phi R_{23}^{(1)} \\
y_{\mathrm{C}} & =\left(R_{13}-R_{12}^{(1)}\right) \cos \phi+R_{12}^{(1)} \sin \alpha / 2 .
\end{aligned}
$$

\section{APPENDIX 2}

In this Appendix, expressions for the volume fractions are derived for a system of grains of equal size with contacts between two or between three grains. The geometry of a three-grain intersection is shown in Figure 3b. It has features in common with the two-grain intersection shown in Figure $3 \mathrm{a}$ and corresponding points are given the same letters. The volume of ice in the three-grain cluster is

$$
V_{\mathrm{i}}=3(V 1-V 2)+V 3-V 4
$$

where $V 1$ is half the volume of ice in a pair of grains with one two-grain contact, $V 2$ is a $60^{\circ}$ segment of a sphere of radius $R_{13}$, the components of $V 3$ fit together to make half a hexagonal prism with spherical caps, and $V 4$ is the volume of the vein running through the centre of the three-grain cluster. Thus, using the notation of Appendix 1,

$$
V 1=2 \mathrm{VZN}-(\mathrm{VZB}-\mathrm{VCB})
$$

and

$$
V 2=\mathrm{VZN} / 3 .
$$

The radius of the inscribed circle in the hexagonal prism is $\delta$ and the height of the prism is $2 y_{\mathrm{C}}$. The spherical caps have radius $R_{13}$ and height $\left(R_{13}-y_{\mathrm{C}}\right)$. Thus,

$$
V 3=6 \delta^{2} y_{\mathrm{C}} \tan \pi / 6+\frac{\pi}{3}\left(R_{13}-y_{\mathrm{C}}\right)^{2}\left(2 R_{13}+y_{\mathrm{C}}\right) .
$$

The volume of the vein may be calculated following Nye (1989), who has shown that the area of the asymptotic cross-section of a water vein at a three-grain intersection in polycrystalline ice is $A\left(r_{\mathrm{V}}\right)^{2}$ where

$$
A=(3)^{\frac{1}{2}} \sin ^{2} \beta+3 / 2 \sin 2 \beta-3 \beta
$$

with $\beta=\pi / 6-\alpha / 2$ and $r_{\mathrm{v}}$ is the radius of the ice-water interface. For the case of a three-grain contact in snow, it is assumed that the cross-sectional area of the vein is constant throughout its length which is $2 y_{\mathrm{C}} \sin \gamma$ where the angle $\gamma$ is given by 


$$
\cos \gamma=(\delta \tan \pi / 6) / y_{\mathrm{C}} .
$$

Thus, the volume of the vein is

$$
V 4=2 y_{\mathrm{C}} \sin \gamma A\left(r_{\mathrm{V}}\right)^{2} .
$$

For the purposes of this paper, it is not necessary to make a separate precise calculation of the volume of a node at each end of the vein. Since the pressure in the vein must be the same as that in the two-grain inclusions which are connected to it and in contact with the same ice grains, the radius of the cylindrical ice-water interfaces of the vein is given by the Laplace equation

$$
\left(\sigma_{12} / r_{\mathrm{v}}\right)=p^{(1)}-p^{(2)}=\sigma_{12}\left(1 / R_{12}^{(1)}+1 / R_{12}^{(2)}\right) .
$$

The volume of water in the three-grain inclusion is

$$
V_{\mathrm{w}}=V 4+3 V 5
$$

where $V 5$ is a section of the annular two-grain water inclusion.

$$
V 5=(2 \pi-2 \gamma)(\mathrm{VHB}-\mathrm{VCB}) .
$$

Let the number of two- and three-grain contacts per grain be $n_{1}$ and $n_{2}$, respectively, arranged in such a way that the inclusions do not interfere. The coordination number $n_{3}=n_{1}+2 n_{2}$.

Then the specific volume of ice is

$$
\phi^{(1)}=N\left(2 \mathrm{VZN}-n_{1}(\mathrm{VZB}-\mathrm{VCB})-n_{2}\left(2 \mathrm{VZN}-V_{\mathrm{i}} / 3\right)\right)
$$

and the specific volume of water is

$$
\phi^{(2)}=N\left(n_{1}(\mathrm{VHB}-\mathrm{VCB})+n_{2} V_{\mathrm{w}} / 3\right) .
$$

MS. received 17 September 1988 and in revised form 6 March 1990 